\title{
Detecting Non-Abelian Statistics with an Electronic Mach-Zehnder Interferometer
}

\author{
D. E. Feldman ${ }^{1}$ and Alexei Kitaev ${ }^{2,3}$ \\ ${ }^{1}$ Department of Physics, Brown University, Providence, Rhode Island 02912, USA \\ ${ }^{2}$ California Institute of Technology, Pasadena, California 91125, USA \\ ${ }^{3}$ Microsoft Project Q, University of California, Santa Barbara, California 93106, USA
}

(Received 21 July 2006; published 3 November 2006)

\begin{abstract}
Fractionally charged quasiparticles in the quantum Hall state with a filling factor $\nu=5 / 2$ are expected to obey non-Abelian statistics. We demonstrate that their statistics can be probed by transport measurements in an electronic Mach-Zehnder interferometer. The tunneling current through the interferometer exhibits a characteristic dependence on the magnetic flux and a nonanalytic dependence on the tunneling amplitudes which can be controlled by gate voltages.
\end{abstract}

One of the central features of the quantum Hall effect (QHE) is the fractional charge and statistics of quasiparticles. The quantum state of bosons or fermions does not change when one particle makes a full turn around another. On the other hand, Laughlin quasiparticles pick up nontrivial phases when they encircle each other. Non-Abelian statistics predicted in some QHE systems [1,2] is even more interesting: The state vector changes its direction in the Hilbert space after a particle makes a full circle.

Shot noise experiments [3] allowed the observation of fractional charges in QHE liquids. Probing fractional statistics is more difficult. It was argued that the mutual statistics of nonidentical quasiparticles was detected in a recent experiment [4] by Camino et al.; however, the interpretation of the experimental results remains controversial $[5,6]$. There are several theoretical proposals for observing the statistics of identical Abelian quasiparticles [7-10] but none of them has been realized experimentally.

Detecting non-Abelian anyons is of special interest due to their promise for fault-tolerant quantum computation [11]. One approach for their observation and probing their statistics is based on current noise in complex geometries $[12,13]$. A simpler proposal involves current through an Aharonov-Bohm interferometer with trapped quasiparticles $[14,15]$. This method should work if the number of trapped quasiparticles does not fluctuate on the measurement time scale [9]. Such a condition might be difficult to satisfy in non-Abelian systems, where the excitation gap is relatively low [16].

We suggest another method, which is free from this limitation. It uses the electronic Mach-Zehnder interferometer recently designed at the Weizmann Institute [17]; see Fig. 1(a). We consider the $\nu=5 / 2$ QHE state and show that the tunneling current $I$ through the interferometer contains signatures of non-Abelian statistics. The current (8) is a periodic function of the magnetic flux $\Phi$ through the interferometer with period $\Phi_{0}=h c / e$, but, in contrast with the Abelian case [10], it is not a simple sine wave. Note the nonanalytic dependence on the interedge tunneling amplitudes $\Gamma_{1}$ and $\Gamma_{2}$ at the quantum point contacts (constrictions) QPC1 and QPC2. In the limit of $\Gamma_{2} \ll \Gamma_{1}$, the formula for the current assumes a sinusoidal form, $I=I_{0}+I_{\phi} \cos \left(2 \pi \Phi / \Phi_{0}+\right.$ const $)$, where the fluxdependent and flux-independent terms are related by the scaling law:

$$
I_{\Phi}\left(\Gamma_{1}, \Gamma_{2}\right) \sim\left[I_{0}\left(\Gamma_{1}, \Gamma_{2}\right)-I_{0}\left(\Gamma_{1}, 0\right)\right]^{b},
$$

with $b=2$. This can be compared with the case of Fermi statistics, where $b=1 / 2$; for Abelian anyons, $b=m+$ $1 / 2$ with an integer $m>0$ [10].

The Letter is organized as follows. First, we briefly discuss the relevant properties of the $\nu=5 / 2$ QHE state and the structure of the Mach-Zehnder interferometer. Next, we derive the above results using a kinetic equation. Finally, we show how to obtain such an equation for an arbitrary non-Abelian state.

Elementary excitations in the $\nu=5 / 2$ liquid carry charge $\pm e / 4$. Because of the non-Abelian statistics, the state of a system of several quasiparticles is not uniquely determined by the quasiparticle coordinates. It is convenient to classify the states according to their superselection
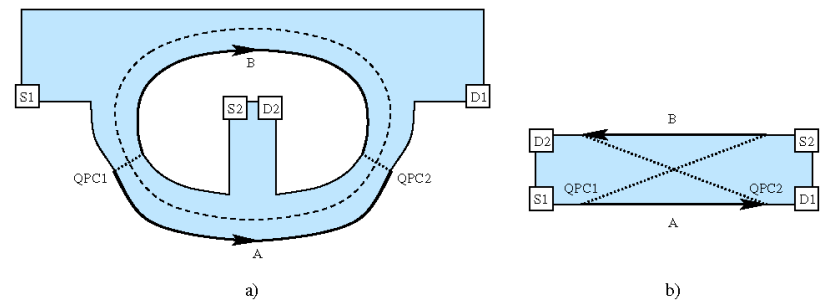

FIG. 1 (color online). (a) Schematic picture of the MachZehnder interferometer: S1, S2, D1, D2 denote sources and drains; arrows show the edge mode propagation direction; quasiparticles tunnel between the edges at quantum point contacts QPC1 and QPC2. (b) Hypothetical setup for the calculation of normalized tunneling rates. 
sectors. (For a review of this formalism, see Appendix $\mathrm{E}$ in Ref. [18].) A superselection sector is characterized by the electric charge $q=n e / 4$ as well as the "topological charge" taking on three values [15]: 1 ("vacuum"), $\epsilon$ ("fermion"), and $\sigma$ ("vortex"). If $n$ is even, then the topological charge can be either 1 or $\epsilon$; if $n$ is odd, then the topological charge is $\sigma$. The topological charge obeys these fusion rules which allow the calculation of the topological charge of the composite system from the charges of its parts: $\epsilon \times \epsilon=1, \epsilon \times \sigma=\sigma, \sigma \times \sigma=1+\epsilon$.

When a $+e / 4$ quasiparticle encircles a composite excitation in the sector $a=(n e / 4, \alpha)$ (where $\alpha$ is the topological charge), it picks up some statistical phase, which depends not only on $n$ and $\alpha$ but also on the topological charge $\beta$ of the whole system. We denote this phase by $\phi_{a b}$, where $b=((n+1) e / 4, \beta)$. It is given by the formula

$$
\phi_{a b}=n \pi / 4+\phi_{\alpha \beta}^{\prime},
$$

where the non-Abelian part $\phi_{\alpha \beta}^{\prime}$ equals 0 if the excitation is in the vacuum sector, $\pi$ if it is in the $\epsilon$ sector, $-\pi / 4$ if the whole system in the vacuum sector, and $3 \pi / 4$ if the whole system is in the $\epsilon$ sector. (In all four cases, the other topological charge is $\sigma$.) We will see that these phases determine the current through the Mach-Zehnder interferometer.

The interferometer [17] is sketched in Fig. 1(a). Charge propagates along two chiral edges in the direction shown by arrows. Quasiparticles tunnel between the edges at the point contacts QPC1 and QPC2. We are interested in the tunneling current between the edges (the current from source S1 to drain D2). It depends on the voltage $V$ between the edges (i.e., the difference of the electrochemical potentials between sources $\mathrm{S} 1$ and $\mathrm{S} 2$ ) and the magnetic flux $\Phi$ through the loop A-QPC2-B-QPC1-A. This loop is defined so as not to touch the leads, because we assume that the leads fully absorb edge excitations. We also assume that the tunneling amplitudes $\Gamma_{1}$ and $\Gamma_{2}$ are small; therefore, we can use perturbation theory. In physical terms, individual tunneling events are regarded as independent and assigned certain probabilities, which are calculated below. When a $+e / 4$ quasiparticle tunnels from the outer edge to the inner edge (through QPC1 or QPC2), the electric charge on the inner edge increases by $e / 4$ (cf. Ref. [10]), and the topological charge changes according to the fusion rules [19]. Specifically, the initial value of the topological charge fuses with $\sigma$ : If the initial charge is 1 or $\epsilon$, then the final charge is $\sigma$; if the initial charge is $\sigma$, then the final charge is 1 or $\epsilon$. In the latter case, the two fusion outcomes occur with equal probabilities: $P_{\sigma \rightarrow 1}^{+}=P_{\sigma \rightarrow \epsilon}^{+}=$ $1 / 2$. Indeed, the tunneling process is independent of the global edge state (due to the absorbing properties of the leads); hence, we may assume that the fusing charges come from uncorrelated sources: The inner edge forms a topologically neutral object with the outer edge, and the tunneling quasiparticle is part of a particle-antiparticle pair created from the vacuum. The probabilities are calculated by applying a topological charge operator of the subsystem (inner edge + tunneling quasiparticle) to the four-body state described above as discussed in Refs. [18,20]. A similar argument applies to $-e / 4$ quasiparticles or, equivalently, to the tunneling of $+e / 4$ quasiparticles from the inner to the outer edge. We will use the notation $P_{a \rightarrow b}^{-}$in this case, though the superscript turns out to be redundant. To summarize, $P_{1 \rightarrow \sigma}^{ \pm}=P_{\epsilon \rightarrow \sigma}^{ \pm}=1, P_{\sigma \rightarrow 1}^{ \pm}=P_{\sigma \rightarrow \epsilon}^{ \pm}=1 / 2$, all other probabilities being zero.

Since bulk excitations are gapped, the low-energy physics is determined by edges [21]. Thus, the Hamiltonian is

$$
\hat{H}=\hat{H}_{\text {edge }}+\left[e^{-i \omega t}\left(\Gamma_{1} \hat{X}_{1}+\Gamma_{2} \hat{X}_{2}\right)+\text { H.c. }\right],
$$

where $\hat{H}_{\text {edge }}$ is the Hamiltonians of the two edges (which carry opposite topological charges but are otherwise independent), $\omega=\mathrm{eV} / 4 \hbar$ describes the voltage bias (cf. Ref. [10]), and the operators $\hat{X}_{1}$ and $\hat{X}_{2}$ correspond to the $e / 4$ charge transfer from the outer to the inner edge at QPC1 and QPC2, respectively. The forward and backward tunneling rates $w^{+}$and $w^{-}$can be calculated in the second order of perturbation theory. It is convenient to consider first the hypothetical setup shown in Fig. 1(b), where the current is independent of the topological charges and the magnetic flux. This yields an expression of the form $\sum_{j, k} r_{j k}^{ \pm} \Gamma_{j}^{*} \Gamma_{k}$. In the real problem, the rates also depend on the superselection sector of the inner edge in the initial and final states [22]. For example,

$$
r_{12}^{+}(a \rightarrow b)=\hbar^{-2} \int_{-\infty}^{+\infty}\left\langle X_{1}^{\dagger}(t) \Pi_{b} X_{2}(0)\right\rangle_{a} e^{i \omega t} d t,
$$

where $\langle\cdots\rangle_{a}$ denotes the thermodynamic average restricted to the superselection sector $a$, and $\Pi_{b}$ is the projector onto the sector $b$. The integrand has the meaning of a particle tunneling through QPC2 and returning via QPC1; hence, it incorporates both the Aharonov-Bohm phase due to the magnetic field $\phi_{\mathrm{mag}}=2 \pi \Phi /\left(4 \Phi_{0}\right)$ and the statistical phase $\phi_{a b}$, Eq. (2). It also includes the fusion probability $P_{a \rightarrow b}^{+}$. Summing over the four possible paths, we obtain this result: $w_{a \rightarrow b}^{ \pm}=P_{a \rightarrow b}^{ \pm} u_{a \rightarrow b}^{ \pm}$, where

$$
u_{a \rightarrow b}^{+}=r_{11}^{+}\left(\left|\Gamma_{1}\right|^{2}+\left|\Gamma_{2}\right|^{2}\right)+\left(r_{12}^{+} e^{i \phi_{\operatorname{mag}}} e^{i \phi_{a b}} \Gamma_{1}^{*} \Gamma_{2}+\text { c.c. }\right) \text {. }
$$

The back tunneling rate can be obtained from the detailed balance principle $u_{b \rightarrow a}^{-}=\exp \left[-e V /\left(4 k_{B} T\right)\right] u_{a \rightarrow b}^{+}$. The fusion probabilities $P_{a \rightarrow b}^{+}$also satisfy a detailed balance equation; see Eq. (10). The constants $r_{11}^{+}$and $r_{12}^{+}$in (5) depend on the expressions for the operators $\hat{H}_{\text {edge }}, \hat{X}_{1}$, and $\hat{X}_{2}$, as well as on the temperature $T$ and the voltage $V$. They are independent of the magnetic flux through the interferometer and the charge labels $a$ and $b$ and could be calculated using the simplified geometry of Fig. 1(b). A calculation based on Wen's hydrodynamic model [23] will be published elsewhere. In this Letter, we discuss 
those properties which are not sensitive to edge physics details but rather to the fractional statistics of tunneling particles.

The statistical phase factor $\exp \left(i \phi_{a b}\right)$ [Eq. (2)] is invariant under the fusion of both $a=(n e / 4, \alpha)$ and $b=((n+$ 1) $e / 4, \beta)$ with an electron, whose superselection sector is $(e, \epsilon)$. Thus, the superselection sectors form 6 equivalence classes, which are characterized by $(n \bmod 4)$ and the choice of 1 or $\epsilon$ if $n$ is even. These classes and possible transitions between them are shown in Fig. 2. At zero temperature (and positive $V$ ), the transitions occur only in the direction of the arrows, since quasiparticles must tunnel from the edge with higher potential to the edge with lower potential. At finite temperatures, transitions between the states connected by the lines in Fig. 2 occur in both directions.

The average tunneling current is given by the equation

$$
I=\frac{e}{4} \sum_{a b} f_{a}\left(w_{a \rightarrow b}^{+}-w_{a \rightarrow b}^{-}\right),
$$

where the distribution function $f$ can be found from the steady state condition

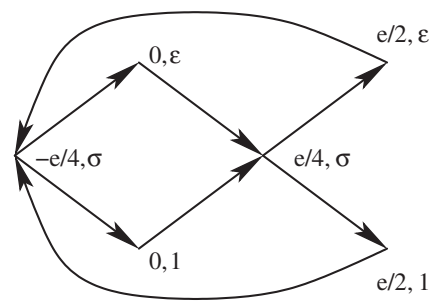

FIG. 2. Six states of the interferometer labeled by the electric and topological charges on the inner edge. Lines show possible transitions. At zero temperature, the transitions occur only in the direction of the arrows.

$$
\frac{d f_{a}}{d t}=\sum_{b}\left[f_{b}\left(w_{b \rightarrow a}^{+}+w_{b \rightarrow a}^{-}\right)-f_{a}\left(w_{a \rightarrow b}^{+}+w_{a \rightarrow b}^{-}\right)\right]=0 .
$$

The solution of the system of linear equations (7) is tedious but straightforward. The general expression for the current is lengthy and will be published elsewhere. It simplifies at zero temperature:

$$
I=\frac{e}{4} r_{11}^{+}\left[\left|\Gamma_{1}\right|^{2}+\left|\Gamma_{2}\right|^{2}\right] \frac{1-\lambda^{2}+\frac{\lambda^{4}}{8}\left[1-\cos \left(2 \pi \Phi / \Phi_{0}+\delta\right)\right]}{1-\frac{3}{4} \lambda^{2}+\frac{\lambda^{4}}{16}\left[1-\cos \left(2 \pi \Phi / \Phi_{0}+\delta\right)-\sin \left(2 \pi \Phi / \Phi_{0}+\delta\right)\right]},
$$

where $\lambda=\left|r_{12}^{+} / r_{11}^{+}\right| 2\left|\Gamma_{1} \Gamma_{2}\right| /\left(\left|\Gamma_{1}\right|^{2}+\left|\Gamma_{2}\right|^{2}\right)$ and $\delta=$ $4 \arg \left(r_{12}^{+} \Gamma_{1}^{*} \Gamma_{2}\right)$. The current is the ratio of two linear trigonometric polynomials of $2 \pi \Phi / \Phi_{0}$. In Abelian quantum Hall states, the current is a sine wave plus a constant [10]. Thus, if the experimentally measured dependence of the tunneling current on the magnetic field at fixed voltage can be fitted by an equation of the form (8), this proves nonAbelian statistics of elementary excitations.

The current is a periodic function of the magnetic flux with period $\Phi_{0}$. This agrees with the Byers-Yang theorem [24], which applies to any interferometer with the magnetic flux passing through a hole.

The dependence of the current on the tunneling amplitudes is nonanalytic. If $\Gamma_{2} \ll \Gamma_{1}$, then the current (8) can be expanded in powers of $\Gamma_{2}$. This gives Eq. (1), which can be used for another experimental test of non-Abelian statistics. The tunneling amplitudes are controlled by gate voltages. At fixed values of the gate voltages, one can measure the magnetic field dependence of the current and extract the flux-dependent and flux-independent contributions as $I_{0}=\left[\max _{\Phi} I(\Phi)+\min _{\Phi} I(\Phi)\right] / 2$ and $I_{\Phi}=$ $\left[\max _{\Phi} I(\Phi)-\min _{\Phi} I(\Phi)\right] / 2$. Changing the gate voltages will allow the testing of the scaling relation (1). Equation (1), however, would have the same form in the (rather unlikely) situation where a quasiparticle picks up an Abelian phase of $\pi / 2$ after a full circle around another quasiparticle. On the other hand, the flux dependence (8) emerges only in the non-Abelian case. In contrast to the Abelian case, the $I-V$ curve is asymmetric for $\nu=5 / 2$ : At
$V<0$, one has to change the overall sign of the current (8) and the sign before $\sin \left(2 \pi \Phi / \Phi_{0}+\delta\right)$ in the denominator.

So far, we have ignored the possibility of a quasiparticle trapping inside the interferometer. Quasiparticles can tunnel not only between the edges but also to or from localized states in the electron liquid. In the usual interferometer geometry $[14,15]$, such tunneling events suppress the interference picture. Indeed, the current through the interferometer depends on the topological charge between the contacts. Each tunneling event between an edge and a localized state changes the topological charge. After averaging with respect to the fluctuating charge, the interference picture disappears. On the other hand, tunneling to localized states plays little role in the Mach-Zehnder interferometer as long as the typical interval between such events exceeds the time between two consecutive tunneling events at QPC1 and QPC2. Indeed, any localized charges may be attributed to either the inner edge or the outer edge depending on their position relative to the interference loop. Hence, one can still use Eqs. (6) and (7), for the calculation of the average current, provided those charges are fixed.

Algebraic theory of anyons [18] allows the calculation of the tunneling rates $w_{a \rightarrow b}^{ \pm}$for an arbitrary type of nonAbelian statistics. As discussed above, the calculation of those rates reduces to the calculation of the statistical phase factors $\exp \left(i \phi_{a b}\right)$ and the fusion probabilities $P_{a \rightarrow b}^{ \pm}$. These are given by the following formulas: 
TABLE I. Statistics in the Pfaffian state.

\begin{tabular}{cccc}
\hline \hline Topological charge & Electric charge & $d_{a}$ & $\theta_{a}$ \\
\hline 1 & $m e / 2$ & 1 & $\exp \left(i \pi m^{2} / 2\right)$ \\
$\epsilon$ & $m e / 2$ & 1 & $-\exp \left(i \pi m^{2} / 2\right)$ \\
$\sigma$ & $e / 4+m e / 2$ & $\sqrt{2}$ & $\exp \left(i \pi\left[2 m^{2}+2 m+1\right] / 4\right)$ \\
\hline \hline
\end{tabular}

$$
\exp \left(i \phi_{a b}\right)=\frac{\theta_{b}}{\theta_{a} \theta_{x}}, \quad P_{a \rightarrow b}^{+}=N_{a x}^{b} \frac{d_{b}}{d_{a} d_{x}},
$$

where $x$ refers to the tunneling quasiparticle, $\theta_{x}$ and $d_{x}$ are the topological spin and the quantum dimension, respectively, and $N_{a x}^{b}$ is the fusion multiplicity. The fusion probability $P_{a \rightarrow b}^{-}$is obtained by replacing $x$ with its antiparticle $\bar{x}$. Note the detailed balance equation:

$$
d_{b}^{2} P_{b \rightarrow a}^{-}=d_{a}^{2} P_{a \rightarrow b}^{+} .
$$

In the $\nu=5 / 2$ Pfaffian state, all fusion multiplicities are equal to 1 , and the quantum dimensions and topological spins can be found in Table I (cf. Ref. [18]). Using that table, one can reproduce the above results for the tunneling probabilities.

In conclusion, we have calculated the tunneling current through the Mach-Zehnder interferometer for the $\nu=5 / 2$ QHE liquid. The dependence of the current on the magnetic flux and tunneling amplitudes can be used for probing non-Abelian statistics.

We thank K.T. Law for the help with figures. A.K. acknowledges support by ARO under Grants No. W911NF-04-1-0236 and No. W911NF-05-1-0294 and by NSF under Grant No. PHY-0456720. D. E. F. acknowledges support by NSF under Grant No. DMR0544116.

[1] G. Moore and N. Read, Nucl. Phys. B360, 362 (1991).

[2] C. Nayak and F. Wilczek, Nucl. Phys. B479, 529 (1996).

[3] R. de Picciotto, M. Reznikov, M. Heiblum, V. Umansky, G. Bunin, and D. Mahalu, Nature (London) 389, 162 (1997); L. Saminadayar, D. C. Glattli, Y. Jin, and B. Etienne, Phys. Rev. Lett. 79, 2526 (1997).

[4] F. E. Camino, W. Zhou, and V. J. Goldman, Phys. Rev. B 72, 075342 (2005).

[5] E.-A. Kim, cond-mat/0604359.

[6] V. J. Goldman, cond-mat/0605614.

[7] C. de C. Chamon, D.E. Freed, S. A. Kivelson, S.L. Sondhi, and X. G. Wen, Phys. Rev. B 55, 2331 (1997).

[8] S. B. Isakov, T. Martin, and S. Ouvry, Phys. Rev. Lett. 83, 580 (1999); I. Safi, P. Devillard, and T. Martin, ibid. 86, 4628 (2001); S. Vishveshwara, ibid. 91, 196803 (2003); E.-A. Kim, M. Lawler, S. Vishveshwara, and E. Fradkin, ibid. 95, 176402 (2005).
[9] C. L. Kane, Phys. Rev. Lett. 90, 226802 (2003).

[10] K. T. Law, D. E. Feldman, and Y. Gefen, Phys. Rev. B 74, 045319 (2006).

[11] A. Yu. Kitaev, Ann. Phys. (N.Y.) 303, 2 (2003).

[12] C. Bena and C. Nayak, Phys. Rev. B 73, 155335 (2006).

[13] E. Grosfeld, S. H. Simon, and A. Stern, Phys. Rev. Lett. 96, 226803 (2006).

[14] A. Stern and B. I. Halperin, Phys. Rev. Lett. 96, 016802 (2006).

[15] P. Bonderson, A. Kitaev, and K. Shtengel, Phys. Rev. Lett. 96, 016803 (2006).

[16] J. S. Xia et al., Phys. Rev. Lett. 93, 176809 (2004); J.P. Eisenstein et al., ibid. 88, 076801 (2002); W. Pan et al., ibid. 83, 3530 (1999); J. P. Eisenstein et al., Surf. Sci. 229, 31 (1990); R. L. Willett et al., Phys. Rev. Lett. 59, 1776 (1987).

[17] Y. Ji, Y. C. Chung, D. Sprinzak, M. Heiblum, D. Mahalu, and H. Shtrikman, Nature (London) 422, 415 (2003).

[18] A. Kitaev, Ann. Phys. (N.Y.) 321, 2 (2006).

[19] The electric charge on each edge is conserved modulo $e$ since electrons can come and go through the leads. The topological charge is not affected by the leads and can be, in principle, measured by transporting a quasiparticle along the dashed line in Fig. 1(a). The charges on the outer edge are always opposite to those on the inner edge (for the topological charge in the Pfaffian state [1] considered in this Letter, "opposite" is the same as "equal").

[20] The relevant degrees of freedom can be characterized by Majorana operators $c_{1}, c_{2}, c_{+}$, and $c_{-}$pertaining to edge 1 (outer), edge 2 (inner), the tunneling particle, and its antiparticle. The initial state $|\Psi\rangle$ is an eigenstate of $i c_{1} c_{2}$ and $i c_{+} c_{-}$, whereas the topological charge in question is described by the operator $i c_{2} c_{+}$(charge 1 corresponds to eigenvalue 1 , and $\epsilon$ corresponds to -1 ). It is easy to see that $\left\langle\Psi\left|i c_{2} c_{+}\right| \Psi\right\rangle=0$; hence, $P_{\sigma \rightarrow 1}=P_{\sigma \rightarrow \epsilon}$.

[21] X.-G. Wen, Quantum Field Theory of Many-Body Systems: From the Origin of Sound to an Origin of Light and Electrons (Oxford University Press, New York, 2004).

[22] We neglect quantum interference between states with different charges on the edges because superpositions of such states decohere faster than the tunneling rate. This is true as long as the second order perturbation theory is valid, which can be demonstrated by deriving the kinetic equation (7) using the Keldysh technique (cf. Ref. [10]).

[23] X.-G. Wen, Adv. Phys. 44, 405 (1995).

[24] N. Byers and C. N. Yang, Phys. Rev. Lett. 7, 46 (1961). 